\title{
A Three-Dimensional Optical Photonic Crystal
}

\author{
Shawn-Yu Lin and J. G. Fleming

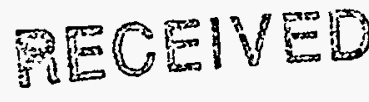 \\ DEC 239998 \\ Sandia National Laboratories, P.O. Box 5800, Albuquerque, NM 87185 (
}

The search for a photonic crystal to confine optical waves in all three dimensions (3D) has proven to be a formidable task. It evolves from an early theoretical suggestion [1,2], a brief skepticism [3-5] and triumph in developing the mm-wave [6-8] and infrared 3D photonic crystals [9]. Yet, the challenge remains, as the ultimate goal for optoelelctronic applications is to realize a 3D crystal at $\lambda \sim 1.5 \mu \mathrm{m}$ communication wavelengths. Operating at visible and near infrared wavelengths, $\lambda=1-2 \mu \mathrm{m}$, a photonic crystal may enhance the spontaneous emission rate $[1,10]$ and give rise to a semiconductor lasers with a zero lasing threshold[11, 12]. Another important application is optically switching, routing and interconnecting light $[13,14]$ with an ultrafast transmission speed of tera-bits per second. A photonic crystal may also serve as a platform for integrating an all-optical circuitry with multiple photonic components, such as waveguides and switches, built on one chip [15]. In this Letter, we report on the successful fabrication of a working 3D crystal operating at optical $\lambda$. The minimum feature size of the 3D structure is 180 nanometers. The 3D crystal is free from defects over the entire 6-inch silicon wafer and has an absolute photonic band gap centered at $\lambda \sim 1.6 \mu \mathrm{m}$. Our data provides the first conclusive evidence for the existence of a full 3D photonic band gap in optical $\lambda$. This development will pave the way to tinier, cheaper, more effective waveguides, optical switches and lasers.

A photonic crystal is the optical analogue of an electronic crystal. It is a periodic 
dielectric structure with its dielectric constant spatially varied according to certain crystal symmetries. Much like electrons in a crystal [16], photonic states inside a photonic crystal are classified into bands and gaps, frequency ranges over which optical waves are allowed or forbidden to propagate respectively. The bands and gaps provide a fundamentally new mechanism for steering and localizing light within a semiconductor chip that is different from that based on conventional optics $[14,15]$. The gap size is determined by the dielectric contrast (or equivalently refractive index) of the two materials that constitute the $3 \mathrm{D}$ structure and by the filling fraction of the higher dielectric material [17]. In our case, the index contrast was $3.6: 1$ and filling fraction $28 \%$.

Although the desired face-centered-cubic (f.c.c.) and diamond crystal structures were proposed a decade ago, attempts to realize it using the novel "three-drilling-holes" approach [18] has not been successful in producing a photonic stop band in optical $\lambda$. Recently, a new approach has emerged. By combining a silicon micromachining technique with a new "layer-stacking" design [17, 19-20], a 3D infrared photonic crystal was fabricated that has a strong stop band at $\lambda=10-14 \mu \mathrm{m}$ [9]. To further increase the operating frequency of a 3D crystal to a communication regime, its minimum feature size must be brought down to a length scale of 100-200 nanometers. To build such a 3D optical photonic crystal, nano-fabrication and precise stacking of nanometer size features are required.

To build a 3D photonic crystal, a layer-by-layer stacking scheme was adopt to construct the diamond lattice structure, see Fig.1(a). This structure has been extensively studied $[17,21]$ and consists of layers of one-dimensional rods with a stacking sequence that repeats itself every four layers. Within each layer the rods are parallel to each other and have a fixed pitch (d). The rod also has a fixed width (w) and height (h). The orientation of 


\section{DISCLAIMER}

This report was prepared as an account of work sponsored by an agency of the United States Government. Neither the United States Government nor any agency thereof, nor any of their employees, make any warranty, express or implied, or assumes any legal liability or responsibility for the accuracy, completeness, or usefulness of any information, apparatus, product, or process disclosed, or represents that its use would not infringe privately owned rights. Reference herein to any specific commercial product, process, or service by trade name, trademark, manufacturer, or otherwise does not necessarily constitute or imply its endorsement, recommendation, or favoring by the United States Government or any agency thereof. The views and opinions of authors expressed herein do not necessarily state or reflect those of the United States Government or any agency thereof. 


\section{DISCLAIMER}

Portions of this document may be illegible in electronic image products. Images are produced from the best available original document. 
the rods on alternate layers is rotated $90^{\circ}$ between layers. Between every other layer, the rods are shifted relative to each other by an amount equal to half the pitch between the rods. For the special case of $4 \mathrm{~h} / \mathrm{d}=1.414$, the lattice can be derived from a f.c.c. unit cell with a basis of two rods. The structure can also be derived by replacing the $<110>$ chain of atoms in the diamond structure with the rods.

The 3D crystals were fabricated from a 6-inch silicon wafer using advanced silicon micro-electro-mechanical systems (MEMS) and integrated circuit (IC) processes. The minimum feature size was the $180-\mathrm{nm}$ width of each of the silicon rods. This is considerably smaller than the minimum feature size of $\sim 0.5 \mu \mathrm{m}$ achievable using our current stepper systems and was achieved using the fillet processing [22]. In a fillet process, the minimum feature size is determined by sidewall coverage of a deposited thin film. Film thickness can be very tightly controlled, and a feature size on the order of $0.18 \mu \mathrm{m}$ is commonly produced. In the first step of the process, a thin film of polysilicon is deposited, photopatterned using the fillet process, and etched. The resulting polysilicon rods are represented by the color red in Fig.1(b). The next step in the processing involves the use of chemical-mechanical-polishing (CMP) to planarize the patterned structure [23]. A layer of silicon dioxide $\left(\mathrm{SiO}_{2}\right)$ shown by the color yellow in Fig.1(b), was deposited in between the lines of polysilicon. The wafers are then polished and planarized using CMP. The planarization step is critical since it prevents the topography generated in the first level from being replicated in the subsequent level. At this point the entire process is repeated to form the subsequent layers of the structure. After completion of the desired number of layers, $\mathrm{SiO}_{2}$ is removed by selective wet etching. A schematic of the final structure is shown in Fig.1(a). 
In Fig.2, scanning electron micrograph (SEM) images of a fabricated five-level structure is shown. The structure, Fig.2a, is periodically stacked like Lincolnlogs, having the top two layers forming right angle crosses. The underlying periodic crosses are also evident. The crystal symmetry axis, $<001>$, is along the layer stacking direction. The rods are aligned either along the $\langle 110\rangle$ or $\langle 1 \overline{1} 0\rangle$ axis. In Fig. 2(b), a SEM cross-sectional view of the same 3D optical crystal is shown. The pitch between adjacent rods is targeted at $\mathrm{d}=0.65 \mu \mathrm{m}$, the rod width $\mathrm{w}=0.18 \mu \mathrm{m}$ and the layer thickness $0.22 \mu \mathrm{m}$. There are slight variations in the pitch between alternated rod pairs. However, the sum of two adjacent pitches is $1.3 \mu \mathrm{m}$.

To prove the existence of a photonic band gap in optical $\lambda$, both transmission and reflection spectra were taken using a Fourier-transform infrared measurement system. Before measurement, the backside of the silicon substrate was polished to a smoothness of better than $0.2 \mu \mathrm{m}$ to avoid significant light scattering. To find the absolute transmittance (T), a transmission spectrum taken from a 3D crystal sample was normalized to that from a bare silicon wafer. This is intended to take into account the slight absorption loss in silicon and reflection loss at silicon/air interfaces. To find the absolute reflectance (R), a reflection spectrum taken from the same 3D crystal is normalized to that of a silver mirror. Our reflectance set-up is capable of taking spectrum over an angular span of $\theta=15^{\circ}-60^{\circ}$ measured from the surface normal, i.e. $<001>$ direction, and has an accuracy of $\pm 5 \%$.

The absolute transmittance of light propagating along the $<001>$ direction, that is, normal to the substrate, of a five-layer 3D crystal is shown in Fig. 3(a). A strong transmittance dip is observed from $\lambda=1.35$ to $2.15 \mu \mathrm{m}$, suggesting the existence of a photonic band gap in optical $\lambda$. The $12 \%$ transmittance at $\lambda=1.55 \mu \mathrm{m}$ is the strongest attenuation, $\sim 10 \mathrm{~dB}$, ever achieved in any 3D photonic crystals in optical $\lambda$. The gap is well defined and extends over a broad spectral range of $\Delta \lambda=0.8 \mu \mathrm{m}$. However, transmittance data alone is not sufficient to prove the existence of a photonic band gap since polysilicon absorption loss may also contribute to a dip in transmittance. A complementary reflectance measurement is therefore 
necessary. A reflectance spectrum taken from the same sample at a slightly tilted angle, $\theta=15^{\circ}$, is shown in Fig.3(b). It is found that detailed line shape of the reflectance data mirrors very well the transmittance over the entire spectrum range. In particular, the reflectance maximum at $\lambda=1.5-1.6 \mu \mathrm{m}$ coincides with the transmittance minimum. This clearly illustrates that, in the band gap regime, backward Bragg reflection dominates, and forward propagation of light is forbidden. More quantitatively, it is found that, at midgap of $\lambda=1.6$ $\mu \mathrm{m}, T=(12 \pm 2) \%$ and $R=(90 \pm 5) \%$, yielding a $T+R=(102 \pm 7) \%[24,25]$. These complementary measurements and the $T+R=1$ relationship provide the first conclusive evidence of the existence of a photonic gap in optical $\lambda$. Indeed, our 3D photonic crystal behaves like a perfect single crystal with negligible, if any, diffraction loss.

To map out dispersion relationship, i.e. energy vs. momentum, of energy bands in the 3D crystal, a tilt-angle transmission measurement was carried out. Light is incident along the plane spanned by $\langle 001\rangle$ and $\langle 110\rangle$ vectors (Fig.1). Equivalently, photon momentum is scanned from crystal symmetry point $\Gamma, \mathrm{K}$ and close to $\mathrm{L}$ in the first Brilliouin Zone (BZ) of the momentum space (see Fig.4). The incident angle $\theta$ was varied from $0^{\circ}$ to $75^{\circ}$ in free space, corresponding to a smaller angle $\theta$ ' as light penetrates into the higher effective index ( $\mathrm{n}_{\text {eff }} \sim 1.73$ ) 3D crystal. In Fig.4, the measured band edge energy is plotted as a function of $\theta$ as solid dots. The upper band edge shifts to higher energies initially, reaches a maximum at $\theta \sim 35^{\circ}$, or $\theta^{\prime} \sim 19.3^{\circ}$, spilits into two bands and eventually tapers off at $\theta \sim$ $75^{\circ}$, or $\theta^{\prime} \sim 33.9^{\circ}$. Simultaneously, the lower band edge shifts toward higher energies and again saturates at $\theta \sim 75^{\circ}$. The saturation is consistent with the fact that, at $\theta^{\prime} \sim 32.5^{\circ}$, photon momentum is approaching the BZ edge, in this case the $\Gamma$-point $\left(\theta^{\prime} \sim 45^{\circ}\right)$. The turning point at $\theta^{\prime} \sim 19.3^{\circ}$ corresponds to the symmetry-point $\mathrm{K}\left(\theta^{\prime} \sim 19.6^{\circ}\right)$, at which photonic states are no longer degenerate, and band edge starts to split. Despite the shifting and splitting in band edges, a universal photonic band gap still exists over a large bandwidth of $\Delta \lambda=$ $450 \mathrm{~nm}$, as defined by the lower band edge at K-point and upper band edge at L-point [21]. Such a large universal band gap, or sometimes called an absolute band gap, warranties the 
strong 3D confinement of light within the photonic crystal.

It is this absolute gap in the optical $\lambda$ that holds promise for a whole new class of very exciting quantum optical devices, which include miniature waveguides, tera-Hertz optical switches, high-Q resonant cavities, and single-mode light-emitting-diodes. Furthemore, since this advance employs silicon technology, the infrastructure to drive this communications revolution is already in place and will help to mate photonics and electronics through the use of a common building block. 


\section{References}

1. E. Yablonovitch, Inhibited spontaneous emission in solid state physics and electronics. Phys. Rev. Lett. 58, 2059-2062 (1987).

2. S. John, Strong localization of photons in certain disordered dielectric superlattices. Phys. Rev. Lett. 58, 2486-2489 (1987).

3. Maddox J., Photonic band gaps bite the dust. Nature 328, 481 (1990).

4. Leung K.M. \& Lu Y.F., Full vector wave calculation of photonic bandgap structures in faced-centered-cubic dielectric medium. Phys. Rev. Lett. 65, 2646-2649 (1990).

5. Zhang Z. \& Satpathy S., Electromagnetic wave propagation in periodic structures: Bloch wave solution of Maxwell equations. Phys. Rev. Lett. 65, 2650-2653 (1990).

6. Pendry J.B., Hopes for photonic band gaps. Nature Vol 351, 354, 435 (1991).

7. Ho K.M., et al., Existence of a photonic gap in periodic dielectric structures. Phys. Rev. Lett. 65, 3152-3155 (1990).

8. Yablonovitch E., et al., Photonic band structure: the face-centered-cubic case employing non-spherical atoms. Phys. Rev. Lett. 67, 2295-2298 (1991).

9. Lin S.Y., et al., A three-dimension photonic crystal operating at infrared wavelengths. Nature, Vol. 394, 251-253 (1998).

10. Purcell E.M, Phys. Rev. 69, 681 (1946).

11. Martini F., et al., Spontaneous and stimulated emission in the thresholdless microlaser. J .Op. Soc. Ame. B10, 360-380 (1993).

12. Deppe D.G. et al., Spontaneous emission from planar microstructures. J . Modern Optics 41, 325-344 (1994).

13. Villeneuve P.R. et al., Single-mode waveguide micro-cavity for fast optical switching. Opt. Lett. 21, 2017-2019 (1996).

14. Lin S.Y. et al., Experimental demonstration of guiding and bending of electromagnetic waves in a photonic crystal. Science 282, 274-277 (1998).

15. Joannopoulos J.D. et al., Photonic crystals: putting a new twist on light. Nature 386, 
143-149 (1997).

16. See, for example, C. Kittel, Introduction to Solid State Physics, Ch.7, p. 183(John Wiley \& Sons, Inc, New York (1980).

17. K.M. Ho et al., Photonic bandgaps in three dimensions: new layer-by-layer periodic structures.Solid State Comm. 89, 413-416 (1994).

18. C.C. Cheng et al., Nano-fabricated three-dimensional photonic crystals operating at optical wavelengths. Physica Scripta, Vol. T68, 17 (1996).

19. Sozuer H. \& Dowling J., Photonic band calculations for woodpile structures. J. Modern Optics, vol. 41, 231-239 (1994).

20. S. Fan et al., Design of three-dimensional photonic crystals at submicron lengthscales. Appl. Phys. Lett. 65, 1466-1468 (1994).

21. E. Ozbay et al., Measurement of a three-dimensional photonic bandgap in a crystal structure made of dielectric rods. Phys. Rev. B 50, 1945-1948 (1994).

22. Fleming J.G. \& Lin S.Y., A Three-Dimensional Photonic Crystal with Stop Band between 1.35 and 1.95 Microns. Opt. Lett., Jan. 01, 1999. This approach relies upon the fact that when a thin film of material is deposited over a step and then is subjected to anisotropic reactive ion etching, a thin sliver of material remains along the sides of the step. If the step height is several times greater than the thickness of the thin film deposited, then the width of the fillet will be identical or at least proportional to the film thickness.

23. Patrick W.J. et al., Applications of chemical mechanical polishing to the fabrication of VLSI circuit interconnections. J. Electrochem. Soc. Vol. 138, 1778-1784 (1991).

24. Our observation is consistent with the theory discussed by Sakoda [25]. In his theory, the condition for diffraction is: $d \lambda>\left(n_{e f f}\right)^{-1}$, where $n_{\text {eff }}$ is the effective index of refraction of the structure. Taking $\mathrm{d}=0.65 \mu \mathrm{m}, \lambda=1.6 \mu \mathrm{m}$ and $\mathrm{n}_{\text {eff }}=1.73, d \lambda=0.41$ and $\left(n_{\text {eff }}\right)^{-1}=0.58$. Since $d \lambda<\left(n_{\text {eff }}\right)^{-1}$, no diffraction loss is expected in our sample. We also note that since the $3 \mathrm{D}$ crystal has a very slight dispersion between 
$\theta=0-15^{\circ}$, the comparison between the transmittance and refiectance data is a valid one.

25. K. Sakoda et al., Transmittance and Bragg reflectivity of two-dimensional photonic lattices. Phys. Rev B. 52, 8992-9002 (1995).

Acknowledgments The authors gratefully acknowledge the support of Dr. H. Weaver and the expertise of the Sandia National Laboratories silicon processing team, especially P. Shea, A. Farino, D. Hetherington and B. Smith. The authors also thank Jim Bur for technical assistance. This work was supported by the United States Department of Energy under contract DE-AC04-94AL85000. Sandia is a multiprogram laboratory operated by Sandia Corporation, a Lockheed Martin Company, for the United States Department of Energy.

Correspondence and requests for materials should be addressed to S.Y.L. (e-mail:SLIN@sandia.gov) 


\section{Figure Captions}

Fig.1 Schematic of the process flow used to construct a 3D photonic crystal. (a) Within each layer, silicon rods are photo-patterned and the spaces in between them filled with silicon dioxide. The process is then repeated until reaching the desire number of layers. (b)As a final step of the process, $\mathrm{SiO}_{2}$ is removed by selective wet etching.

Fig.2 SEM images of a released five layer structure. (a) A top view SEM image. The parallel rods are equally spaced within each layer and stacked in a layer by layer fashion according a special sequence that repeats itself every four layers. Rods in the first two layers form right angle crosses. The underlying two layers also form crosses and are shifted diagonally half way. (b) A side view SEM image of the same structure. There are slight variations in the pitch between alternated rod pairs. However, the sum of two adjacent pitches is $1.3 \mu \mathrm{m}$.

Fig.3 (a) A transmission spectrum for the 3D optical photonic crystal as shown in Fig.2. The transmission dip, indicative of the existence of a photonic band gap, is well defined, centered at $\lambda \sim 1.6 \mu \mathrm{m}$ and extends over a broad spectral range, $\Delta \lambda=800 \mathrm{~nm}$. (b) A. reflectance spectrum taken from the same 3D crystal. The reflectance maximum coincides well with the transmittance minimum.

Fig.4 The measured band edge energy of a 3D photonic crystal plotted as a function of $\theta$. The major symmetry points, e.g. $\Gamma, X^{\prime}$ and $\mathrm{K}$, in the first Brillouin zone is also shown in the insert. 


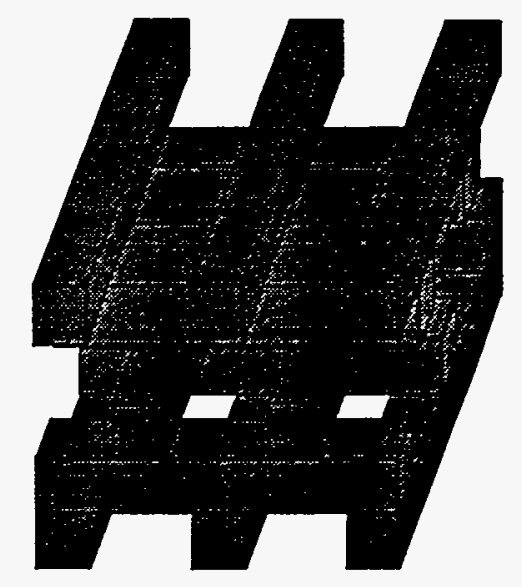

(a)

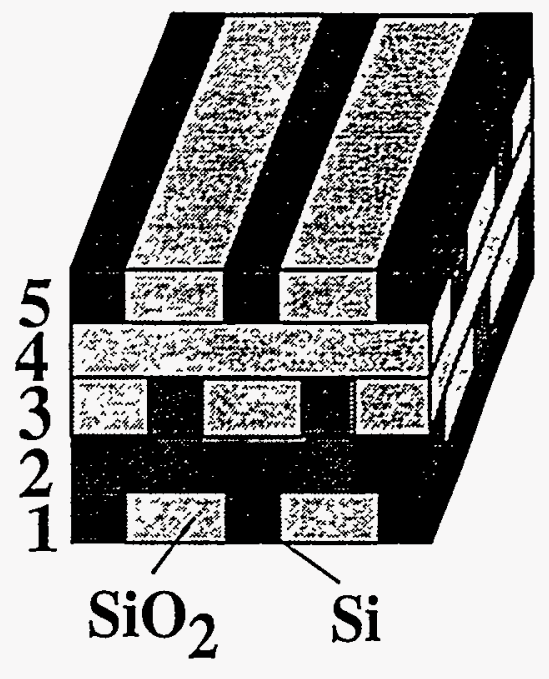

(b)

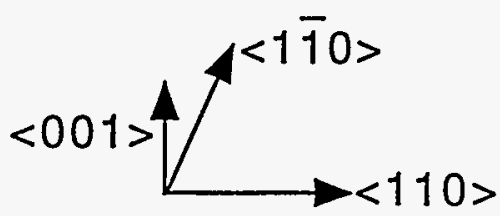

Fig. 1 


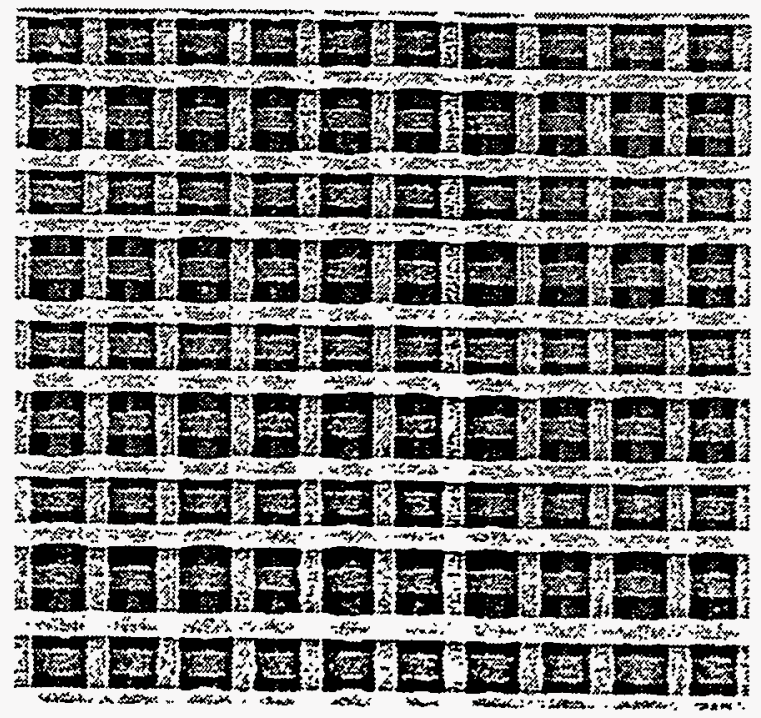

(a)

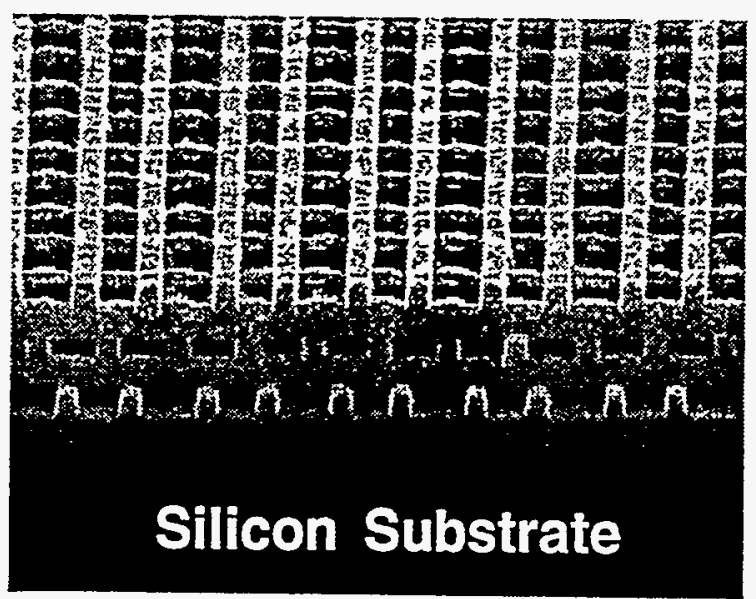

(b) 


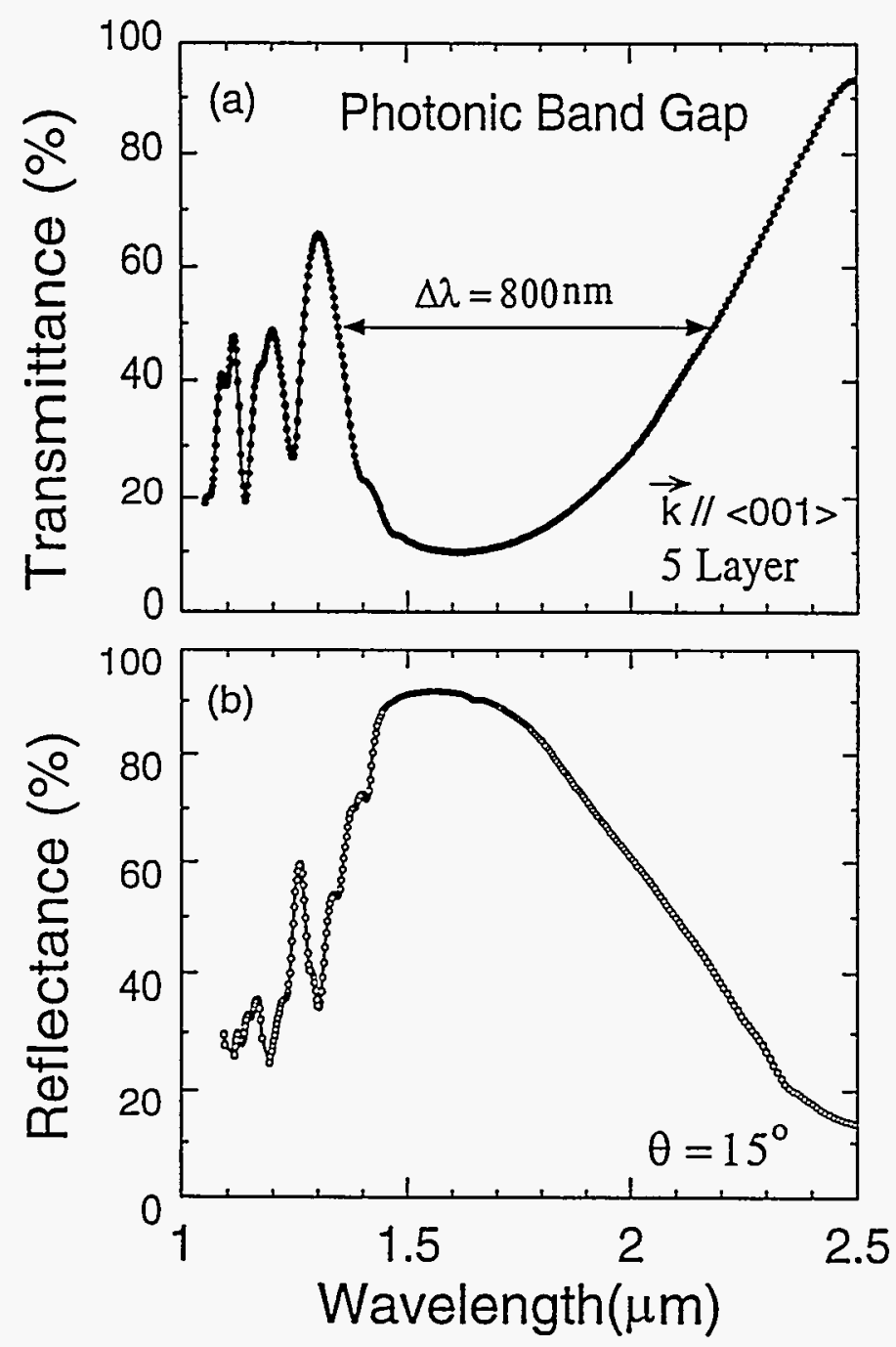

Fig.3 
(a)
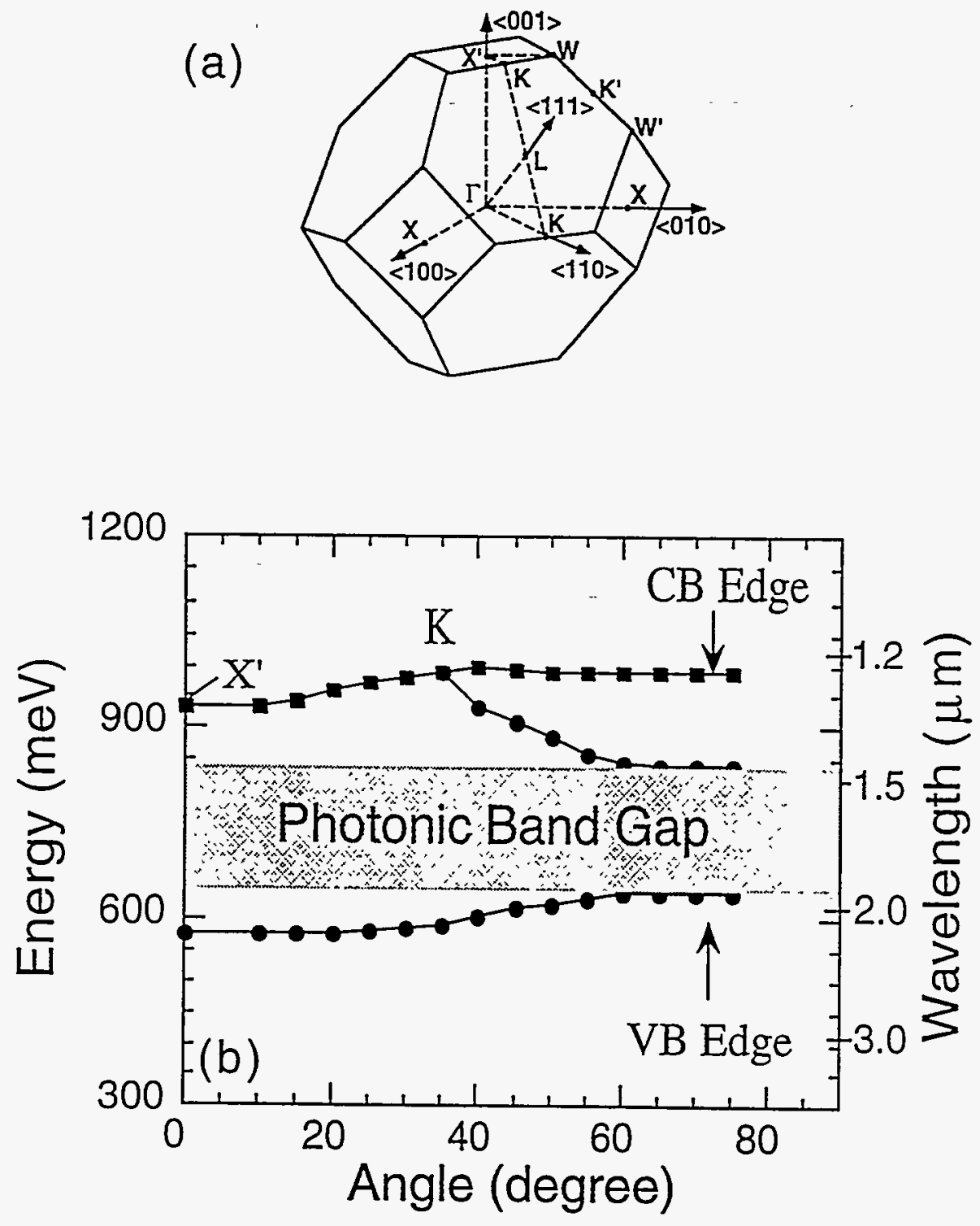